\title{
Development and characterization of human immortalized cell-based in vitro BBB tri-culture model
}

\section{Keita Kitamura ${ }^{1}$, Kenta Umehara ${ }^{1}$, Shota Suzuki ${ }^{1}, K$ Kan Chiba ${ }^{1}$, Naohiko Anzai ${ }^{2}$, Hidetaka Akita ${ }^{1}$, Tomomi Furihata ${ }^{1,2}$}

${ }^{I}$ Grad Sch Pharmaceut Sci, Chiba University, Japan, ${ }^{2}$ Grad Sch Med, Chiba University, Japan

[Introduction] The blood-brain barrier (BBB), which is formed by brain microvascular endothelial cells (BMEC) together with brain pericytes and astrocytes, strictly restricts entry of various drugs into the brain. For evaluation of drug permeability across the BBB in humans, in vitro human BBB model has been considered to be a promising tool. To develop such in vitro human BBB model, immortalized cells carrying both scalability and functionality are expected to be useful, and accordingly we have recently isolated human brain microvascular endothelial cells/ conditionally immortalized, clone 18 (HBMEC/ci18). Therefore, in this study, we aimed to characterize their BBB properties, and also tried to develop an immortalized cell-based BBB tri-culture model.

[Methods] The barrier functions of HBMEC/ci18 were assessed by drug permeability assay and transendothelial electrical resistance (TEER) measurement. The expression of intercellular junction proteins and endothelial marker proteins were examined by immunocytochemistry. For development of an immortalized cell-based BBB tri-culture model, human immortalized astrocytes (HASTR/ci35) and brain pericytes (HBPC/ci37), which we previously established, were cocultured with HBMEC/ci18 using a transwell system.

[Results] BMEC traits of HBMEC/ci18 were confirmed by immunocytochemistry, where CD31, von Willebrand factor, vascular endothelial-cadherin, and zonula occludens-1 expression were clearly observed. Next, we examined the barrier property of the HBMEC/ci18-monolayer BBB model. The results of drug permeability assays showed that, while the Pe value $\left(10^{-3} \mathrm{~cm} / \mathrm{min}\right)$ of $\mathrm{Na}-\mathrm{F}$ was $3.7 \pm 0.4$, those of memantine and caffeine were significantly higher $(42.5 \pm 4.8$ and $47.2 \pm 7.1$, respectively). Finally, in conjunction with HASTR/ci35 and HBPC/ci37, we developed a BBB tri-culture model and tested its barrier properties. The result showed that the TEER value and the lucifer yellow Pe value of tricultured HBMEC/ci18 was higher and lower than those of the mono-culture model (2.0-fold, 0.7-fold, respectively).

[Conclusion] Our results show that HBMEC/ci18 themselves possess BBB properties, but the properties are greatly enhanced when HBMEC/ci18 are co-cultured with HASTR/ci35 and HBPC/ci37. Thus, it can be expected that the immortalized cell-based in vitro human BBB tri-culture model will be a useful tool for prediction of drug permeability in CNS drug development. 